Research Article

\title{
Modelling Mass Transfer Coefficients During Drop Formation
}

\author{
M. A. Hashem* and M. N. Aimaghrabi
}

Chem. Eng. Dept, College of Eng. Gizan University, Kingdom of Saudia Arabia

Received 16 November 2011; Accepted 20 January 2013

\begin{abstract}
A concise review of relevant experimental observations and modelling of mass transfer during drop formation, based on recent studies, is presented. Empirical and theoretical models developed for drop formation are briefly described. Experimental investigations are also performed to explore the mass transfer during drop formation in extraction column and to evaluate the effect of different parameters on the rate of formation. The effects of formation time, which is of industrial relevance, on the mass transfer from drops are discussed. It has been found that the mass transfer coefficients are largest in the initial stages of drop formation when convection is most significant. The resulting data was used to check the available models in the literature. A comparison between the experimental data and the available models in the literature has been made and a modified new correlation was suggested. The results would provide the basic information about the rate of drop formation and design of extraction column, and consequently the environmental impact of solvent extraction processes. Hence, the potential for increasing the reliability of these results is correspondingly increased.
\end{abstract}

Keyword: Solvent extraction, mass transfer, drop formation, modelling

\section{Introduction}

Liquid-liquid extraction plays an important role as a separation technique because of its lower energy cost and more gentle treatment, in comparison with distillation its importance will increase in the future [1,2]. In most industrial extraction equipment one phase is dispersed as a swarm of drops in a second continuous phase. This method of contact has the advantage of providing a large interfacial area per unit volume for the mass transfer [2]. Mass transfer to and from drops moving in a continuous phase is common to many industrial processes especially those involving liquid-liquid extraction and liquid phase reaction [3].

The knowledge of the behaviour of single droplets is the basis for the design of solvent extraction columns $[4,5,6]$. Thus, literature on single droplets can be found in several fields of science and engineering (2). In liquid-liquid extraction, the single-droplet behavior is determined by mass transfer and droplet sedimentation, which take place simultaneously and influence each other. [7, 8, 9]. In recent years much attention has been given to studies of the motion and mass transfer behaviour of single liquid drops. The rationale for this emphasis is that the operating characteristics of equipment in which one liquid phase is dispersed in another should be ultimately determined by what happens to individual droplets. In many industrial operations the flow conditions are turbulent and drop shapes often deviate from spherical, in addition, individual drops may coalesce with other drops or break up into smaller ones. So the study of the motion of liquid drops and their behaviour in another liquid medium of infinite and restricted extent is of importance to the liquid -liquid extraction process since in most liquid - liquid extraction equipment the

*E-mail address: Drmohsen2@hotmail.com

ISSN: 1791-2377 @ 2013 Kavala Institute of Technology. All rights reserved. contact between the liquids is secured through dispersion of the phases as drops [10,11]. Hence knowledge of drop phenomena should provide the basic information needed for the design of most liquid-liquid contactors in which the drop size and velocity are related to the transfer efficiency and the terminal velocity to the capacity of equipment. The problem of predicting transfer rates on a completely theoretical basis in even single drop or bubble situations is fairly complicated, as it involves not only diffusion variables but also those that characterise the hydrodynamic environment $[12,13]$. The latter have hither to defied all attempts at a reasonably complete analysis. In addition to internal turbulence, changes in flow regime around the sphere, oscillations in shape, uneven fluctuations in terminal velocity, eruption effects at the interface, and the ubiquitous presence of trace quantities of surface-active agents (which accounts for a major part of observed random variations) are other stochastic disturbances, which can be studied only at confidence levels [14]. On an experimental level, single fluid sphere study deals separately with three stages of life, namely, (i) the formation stage (in which the drop or bubble is formed at the tip of a nozzle or orifice), (ii) the travel stage (in which it moves in the continuous phase with a gradually increasing velocity which asymptotically approaches a terminal value), and (iii) the coalescence stage (in which it merges into previously collected raffinate). In every stage, the total resistance to transfer is then the sum total of three separate additive resistances; namely, (i) the resistance within the dispersed phase, (ii) the resistance at the interface, and (iii) the resistance within the continuous phase. Separate computations are necessary for each stage. In the present study, only the formation stage is considered. The current literature, Table 1, [15, 16, and 17] of chemical engineering contains a large number of theoretical and experimental investigations reports on mass transfer and analogous heat transfer studies of drop and bubbles in 
various type of columns. Single drop studies have frequently concerned on mass transfer and drop dynamics in the free fall period, with less attention given to formation and coalescence, experimental studies are thus often designed to eliminate the mass transfer in these latter stages or to evaluate it as an end effect by some extrapolation procedure [18]. A recent overview of the experimental techniques to measure the mass transfer rate in liquid-liquid systems can be found in Henschke an Pfennig [4].

\section{Previous drop formation models}

Several investigators have presented theoretical models governing mass transfer and experimental observation revealing quantitative trends, but no accurate means of predicting the dispersed phase mass transfer rate during drop formation have been published. The different models are summarised in Table (1), they are of the same form with different constants for each mechanism.

The models presented in the table are only valid for cases where no extra mass transfer occurs by liquid flow and where eruptions at the interface are not present, which is not always the case. In the models derived not enough attention has been paid to the different rates of drop formation.

\section{Scope of the present work}

The present study is a continuation of our previous work $[16,19,20,21]$ in which the hydrodynamics and mass transfer performance in a extraction column was studied and an effort was made towards contribution for the sum of the data required for extractors design, on the basis of which such column can be better modelled and optimized for design purposes. In this study, the influence of different parameters on the rate of mass transfer during drop formation is presented for the extraction of acetic acid from the aqueous acetic acid solution with toluene as the solvent. The mass transfer from single liquid drop was to be measured during the drop formation period, which terminated upon detachment of the drop from the needle. Comparison of the measured values for the mass transfer and those predicted from the others models has been made to show the validity of single liquid drop data for use in column design work.

\section{System studied}

The system studied was acetic acid/ toluene/ water (continuous phase resistance). The initial concentration of acetic acid ion the continuous phase was $5 \%$ by volume for all experiments. All the chemicals used were of analytical grade reagent and all these chemicals were used as received without further purification. The physical properties [22] of the mutually saturated phases are: $\rho_{\mathrm{c}}=996 \mathrm{~kg} / \mathrm{m} 3, \rho_{\mathrm{d}}=867$ $\mathrm{kg} / \mathrm{m} 3, \mu_{\mathrm{c}}=0.977 \mathrm{~kg} / \mathrm{ms} .103, \mu_{\mathrm{d}}=0.576 \mathrm{~kg} / \mathrm{ms} .103$ and $\gamma=$ $29.3 \mathrm{mN} / \mathrm{m}$. The study was restricted to evaluation of the mass transfer during drop formation only. Mass transfer data were obtained by careful determination of the difference in concentration of acetic acid between the two phases. This difference being the amount of organic plus associated water extract by the drop.

\section{Apparatus and procedure}

The column used, consists of glass tubing, $0.15 \mathrm{~m}$ diameter, $0.5 \mathrm{~m}$ tall and is similar in general design to that described by $[20,21]$. The column diameter was large enough to prevent wall effects. The wall effect calculated using an equation recommended by Grace [22], was found to be negligible.

Table 1: Theoretical models for dispersed phase mass transfer during drop formation

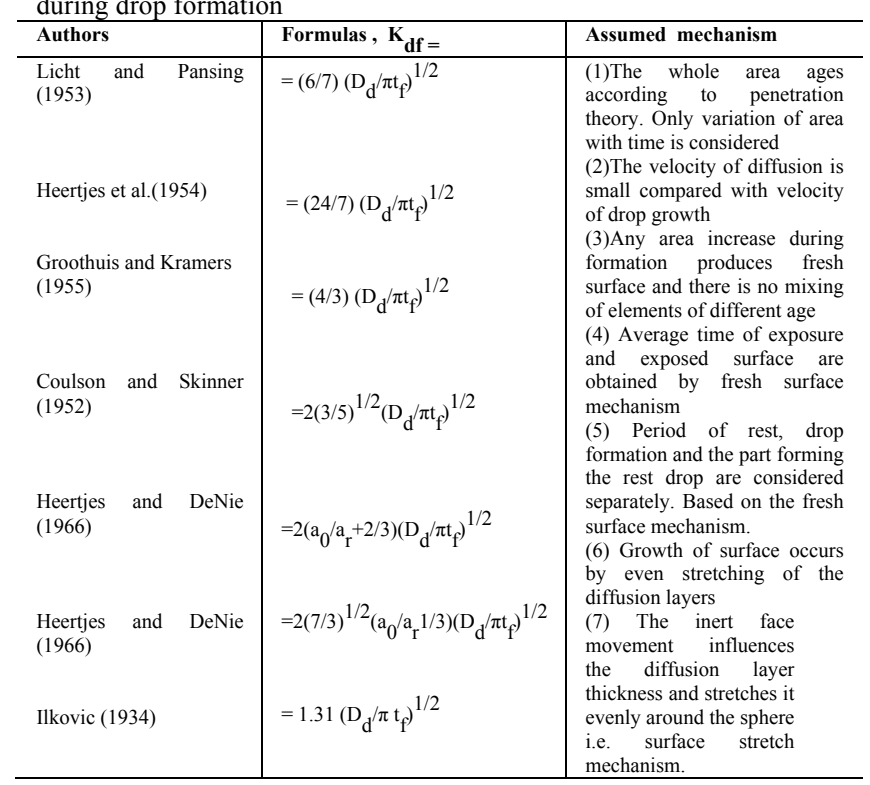

Each series of experiment was carried out with constant conditions. Thus a constant flow rate, constant drop sizes. The column was filled with the aqueous phase (distilled water) before the solvent (dispersed phase) is fed. Rotameter on the lines to and from the column enabled accurate flow settings and adjustment to be made thus ensuring that balanced flows were rapidly achieved and then maintained.

The dispersed drops were formed within water and a positive displacement pump (Razel Scientific Instrument type A-99) was used to introduce the organic phase into the bottom of the column. The syringe pump was kept running while the column was filled with continuous phase water $[13,21]$. The pump and syringe were mounted at a higher level in order to avoid back flow of solvent in the syringe. Great car was taken to avoid the contamination of the apparatus by a foreign substances $[22,23]$. Experiments were performed using different glass needles to obtain drops of different size. The equivalent spherical drop diameter was calculated, knowing the flow rate from the syringe pump and counting the number of drops formed and measuring the time of formation, using a stop watch. At least 300 drops were timed for each run, after a period, which is enough to reach to steady state conditions [16], i.e. constant formation rate and reproducible drops. The nozzle tips were immersed approximately $5 \mathrm{~mm}$ in the continuous phase and the run was initiated by positioning the feed needle valve and starting the stopwatch. The best procedure is to capture drops at a point just above the needle tip where drops are formed [24]. To keep the mass transfer during drop rise from the needle top $(2 \mathrm{~mm})$ as small as possible a small collector was used. The interfacial area in the collector (funnel) was kept small by occasionally pulling the drops into a pipette. The error in measuring the concentration just above the 
needle may be problematical but there may no reasonable alternative procedure [24]. Except other wise mentioned all the experiments were conducted with the aqueous phase continuous and the organic phase dispersed at laboratory ambient temperature $25 \pm 2^{\circ} \mathrm{C}$.

\section{Evaluation of single drop mass transfer data}

Mass transfer rates in two phase systems are caused by driving forces due to thermodynamic non-equilibrium [25]. In contrast to most other separation processes, liquid-liquid extraction is carried out isothermally and under atmospheric pressure. Therefore, only one is driving force, the difference in chemical potentials, remains which can be described as a concentration gradient of a solute from one phase to the other. In general the driving force can be related to the molar flux per unit area.

In order to calculate the mass transfer during the free motion of a drop from the measurable solute concentration, the following model has been derived that leads to an expression for the time-averaged single solute overall mass transfer coefficient $[16,19]$.

$\mathrm{Vd} \mathrm{dC} / \mathrm{dt}=\mathrm{k}$ od $\mathrm{Ad}\left(\mathrm{C}-\mathrm{C}^{*}\right)$

Where the mass transfer coefficient $(\mathrm{k})$ is a constant of proportionality whose reciprocal value describes the mass transfer resistance in the phase considered and by using the specific surface area of a drop (Ad) and its volume (Vd)

$\operatorname{Ad} / \mathrm{Vd}=(\pi \mathrm{d} 2) /(\pi \mathrm{d} 3 / 6)=6 / \mathrm{d}$

As a result of integration, eqn. (1) can now be written as follows:

$\mathrm{k}$ od $=\mathrm{d} / 6 \mathrm{t} \ln \left[\left(\mathrm{Ci}-\mathrm{C}^{*}\right) /\left(\mathrm{Co}-\mathrm{C}^{*}\right)\right]$

The fractional approach to equilibrium is used to relate analytical results to a mass transfer coefficient, i.e

$\mathrm{E}=(\mathrm{Ci}-\mathrm{Co}) /\left(\mathrm{Ci}-\mathrm{C}^{*}\right)$

Where $\mathrm{E}$ is the degree of extraction

Equation (3) simplifies to

$\mathrm{kdf}=-(\mathrm{d} / 6 \mathrm{t}) \operatorname{Ln}(1-\mathrm{E})$

Hence

$\mathrm{kd} \mathrm{d} / \mathrm{D}=\mathrm{Shd}=-\mathrm{d} 2 / 6 \mathrm{D} \mathrm{d}$ tf $\operatorname{Ln}(1-\mathrm{E})$

Analysis of solvent drops after passage through the needle a known time allows mass transfer coefficient to be calculated from the equation:

$(\mathrm{Co}-\mathrm{Ci}) /\left(\mathrm{C}^{*}-\mathrm{Ci}\right)=(1-\exp (-6 \mathrm{kdf} \mathrm{tf} / \mathrm{d}))$

Where $\mathrm{Ci}$ is the inlet and Co outlet dispersed phase concentration, Drop formation time at the needle was easily found by counting drops at a fixed flow rate over a given time. This drop formation was included in the time used for calculating drop diameter. Since there was no solute initially in the water, the drop concentration in equilibrium with the aqueous phase, $\mathrm{C}^{*}=0$. i.e $\mathrm{kdf}=\mathrm{d} / 6 \mathrm{tf} \ln (\mathrm{Ci} / \mathrm{Co})$

A relation between this time-averaged overall dispersed phase mass transfer coefficient and the time variant one, Kod,t is given by:

$\operatorname{Kod}=1 / \Delta \mathrm{t} \int \operatorname{kod}, \mathrm{t} d \mathrm{t}$

\section{Results and Discussions}

7. 1. Effect of continuous phase flow rate

Transfer in the continuous phase surrounding droplets in liquid-liquid system is determined by a combination of molecular diffusion and convection in the continuous phase. In the absence of the agitation, the latter contribution depends on gravitational effects arising from the density difference between the two phases, which indicate relative motion between each drop and its surrounding liquid.

Figure 1 shows the effect of flow rate of the continuous phase on the rate of the mass transfer during drop formation. The coefficient increases with increasing flow rate (i.e. increasing Reynolds number, Figure 2).

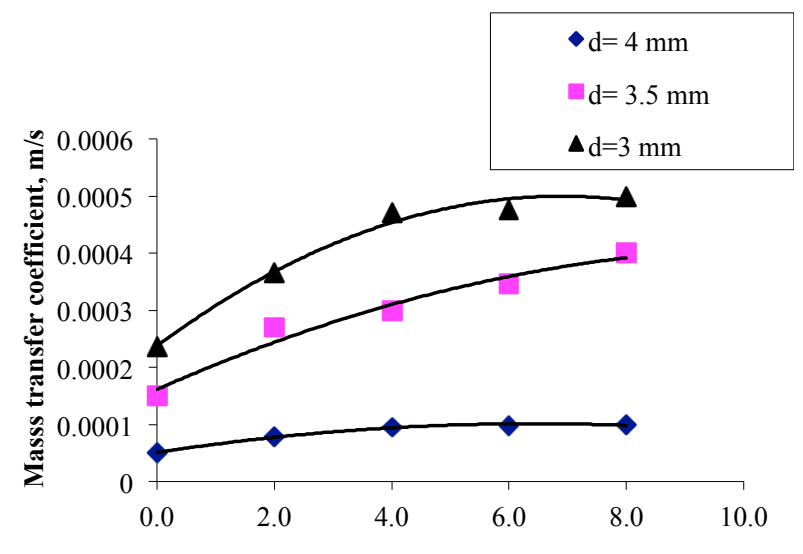

Flow rate of continuous phase, $1 / \mathrm{min}$

Fig.1. Effect of cintinuous phase velocity on the mass transfer coefficient during drop formation

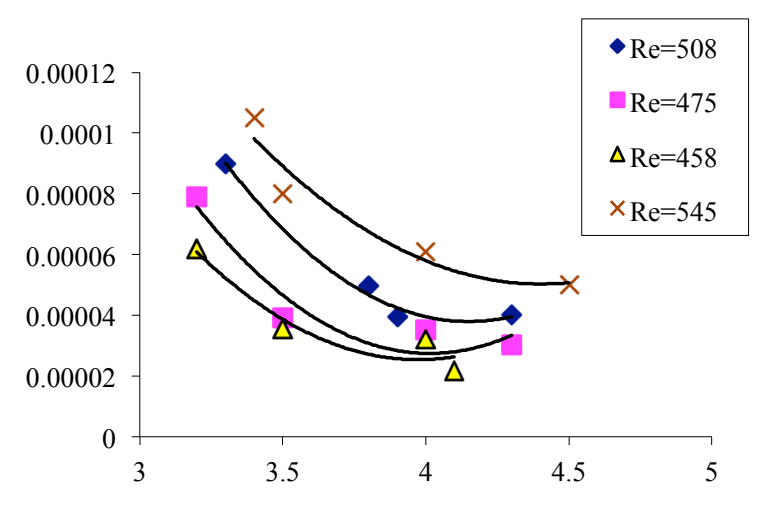

Formation time,s

Fig.2. Mass transfer coefficient at different values of Reynolds number 
It is appear that the continuous phase flow rate is important in determining the mass transfer rate. This circulation has a significant effect on increasing the formation time of drop and hence increasing the drop formation mass transfer. Figure 3

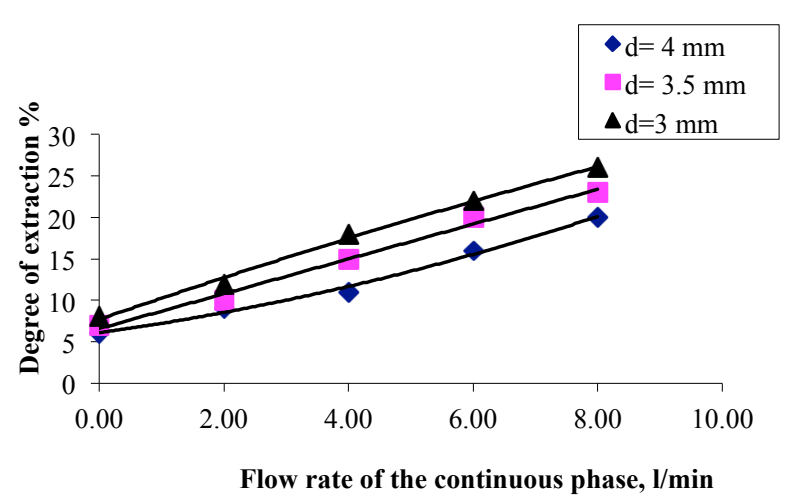

Fig.3. Effect of continuous phase velocity on the degree of extraction during drop formation

show the profile of down flow of the continuous phase against degree of extraction for different drop size. The degree of extraction increases with increasing the down flow rate due to the increase of the continuous phase velocity. Extraction during drop formation before detachment of drops from nozzles is reported to form a major fraction of the total extraction [26]. This marked difference in extraction may be explained by considering, complete internal circulation, partial circulation at the periphery of the drop with the central region of the drop stagnant as in the transient film model and complete rigidity of the drop [27]. The prediction of precise extraction rates during drop formation is difficult because of the rapid changes in interfacial tension and the interfacial area of the droplet which occur during this period. Discrepancies between the results obtained may be attributed to surface-active impurities which form a barrier across the liquid - liquid interface, thereby setting up a direct resistance to diffusion.

\section{2. Effect of dispersed phase flow rate}

The variation of the mass transfer coefficient with formation time is shown in Figure 4.

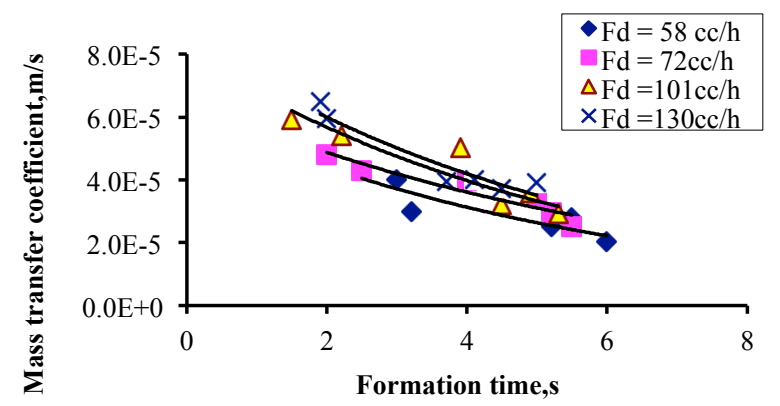

Fig.4. Effect of dispersed phase flow rate on the mass transfer during drop formation, $\mathrm{d}=3 \mathrm{~mm}$

At various formation rate of dispersed phase as parameter. In every case the coefficient is initially large but rapidly decreases. The implication of this behaviour is that the convection generated in the drop by the velocity of fluid leaving the needle exerts a significant influence on the mass transfer and this convection is great when the drop size is small (Figure 5).

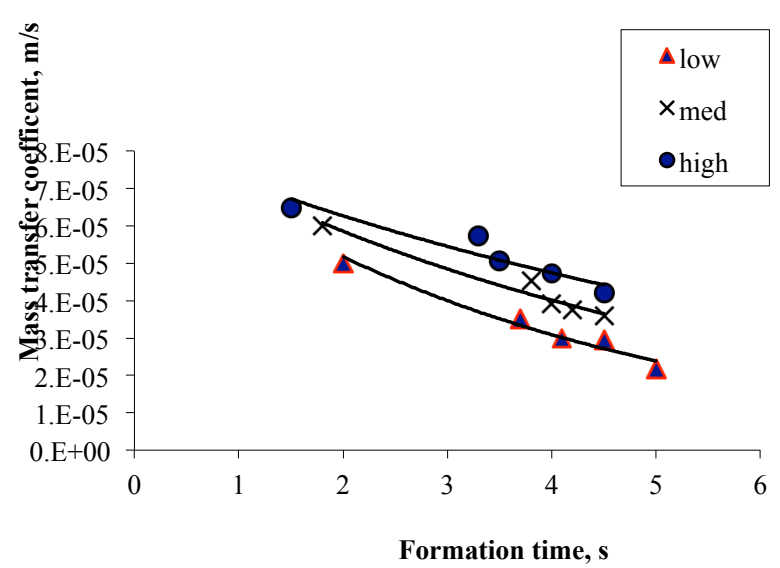

Fig. 5: Effect of dispersed phase rate on MTC at zero down flow of the continuous phase

Since this system is taken to be continuous phase resistance controlled, the effect would have to be transmitted across the interface to the influence the boundary layer around the drop [27]. It is concluded that the dispersed phase flow rate is important as that of the continuous phase in determining the rate of mass transfer.

\subsection{Effect of needle diameter}

Figure 6 shows the effect of needle diameter on the rate of mass transfer during drop formation. Market difference in the rate of mass transfer existed in the rate of transfer from three needles up to a time of formation of at least two seconds. The agitation inside drops formed on the small diameter tube was substantially greater than inside those formed on the wider tube, at least for short formation times, resulting an increase in the rate of transfer At complete formation the mass transfer was about the same for all needle and the rate decrease there after, this is in agreement by observation of many authors [29, 30]. A decrease in the transfer rate during the first second after formation and the drop were internally stagnant after another few seconds of life, supports the view that the drop internal circulation initially observed is induced by the formation process.

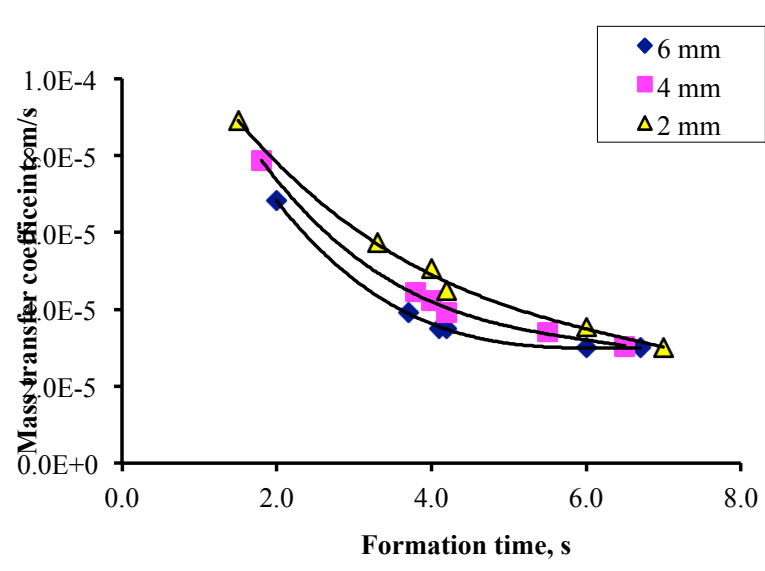

Fig. 6. Effect of needle size on the rate of mass transfer 


\subsection{Modelling of the experimental results}

In attempting to select a model for design use, it would be helpful if some indication could be found regarding whether the surface of a growing drop enlarged by stretching of the existing surface or by the addition of fresh elements. Recent experiments could bear on this question [1].

The different models are of the same form with different constants for each mechanism. The drop is assumed to grow as a sphere and mass transfer during formation is based on the time of formation and the surface area at the point of detachment. These equations are also based on theoretical models of which the following conditions are assumed in the derivations:

a) the interfacial concentration is that at saturation, b) mass transfer is by diffusion at the interface, c) the diffusion process is slow with respect to that of dropgrowthand

d) variation in diffusion coefficient in the flow direction is negligible. Liang and Slater [12] have reviewed much of the work done on mass transfer to forming drops. The degree of extractions can be written as:

$\mathrm{E}=\mathrm{C}(\mathrm{Dd} \mathrm{tf}) 0.5 / \mathrm{d}$

Where the coefficient, $\mathrm{C}$, varies with model assumption [30]. Some of the literature data [12] does not give the expected relationship and no account has been taken of the influence of surfactants [29].Skelland and Minhas [29] tested the theoretical models and reported that the models predict lower mass transfer rate than the observed values. This is because allowance was not made for internal circulation, the effect of interfacial turbulence, the influence of drop rise time and disturbances associated with the drop detachment. They recommended the following empirical model:

Kdf tf /d = 0.0432 (Fr)0.089(d2 / Dd tf )-0.334(Oh)-0.601

With an average absolute deviation of $26 \%$, where $\mathrm{Fr}=$ Froude number and $\mathrm{Oh}=$ Ohnesorge number.

The models presented above are only valid for cases where no addition mass transfer occurs by liquid flow and where eruptions at the interface are not present, which is not always the case. In the models derived not enough attention has been paid to the different rates of drop formation. The models based on formation of fresh surface element give the best prediction of mass transfer during drop formation [30. 31].Humphrey [32] found that the coefficient is high for the initial drop and then rapidly decreases as the drop size begins to increase. The more rapid of this formation have higher initial coefficients but they decay more quickly since the drop volume increasing more rapidly. Suggested that the early rapid fall off corresponds to the damping of convection within the drop and the mass transfer subsequent to this time may then be attributed to molecular diffusion alone. Marsh and Heideger [33] employed a photographic technique to measure the rate of extraction during formation, rise and coalescence and attributed their high mass transfer rate as compared to the theoretical models to intensive circulation within the drops. This liquid motion is probably caused by high velocity of the continuous phase as it enters the drop and decays rapidly after detachment. Then the drop after a brief time becomes either stagnant or slowly circulating under the influence of the relative phase velocity. They concluded that $32 \%$ of transfer occurred during formation, $30 \%$ during the first second after formation and the remaining $38 \%$ during the rest of the drop life. Skelland [31] obtained indirect evidence from experiment that equation is adequate for the prediction of the respective individual mass transfer coefficient during drop formation at the end of the jet for liquid-liquid systems. The correlation was of course originally established for drops forming at a nozzle tip.

In this work, the mass transfer during drop formation was estimated and an empirical correlation of the results was therefore attempted. The following list of variables ought to fully describe the different phenomena which influence the mass transfer during drop formation: formation time, drop diameter, needle diameter, flow rate of the dispersed phase through the needle, flow rate of continuous phase or circulation rate, distribution coefficient of the solute, interfacial tension of the system, both density and viscosity the of continuous and dispersed phase, i.e.

$\mathrm{kdf}=\mathrm{f}(\mathrm{tf}, \mathrm{d}, \mathrm{dn} F d, F c D, \gamma, \mu \mathrm{c} \mu \mathrm{d}, \rho \mathrm{d} \rho \mathrm{c})$

Based on the above discussion, it is clear that circulation of the continuous phase has a significant effect on increasing the time of formation and rate of mass transfer. So by introducing an additional parameter such as flow rate of the continuous and dispersed phase to equation (12) to correlate the data covering down flow up to $81 / \mathrm{min}$ the mass transfer coefficient in the presence and absence of down flow of the continuous phase can be calculated. The recommended correlation is

$\mathrm{kdf}$ tf $/ \mathrm{d}=0.0432(\mathrm{Fr}) 0.089(\mathrm{~d} 2 / \mathrm{Dd}$ tf $)-0.334(\mathrm{Oh})-0.601(1+(\mathrm{Re}) 0,3)$

or

$\mathrm{kdf}=0.0432(\mathrm{Fr}) 0.089(\mathrm{~d} 2 / \mathrm{Dd}$ tf $)-0.334(\mathrm{Oh})-0.601(1+(\mathrm{Re}) 0.03) \mathrm{d} / \mathrm{tf}$

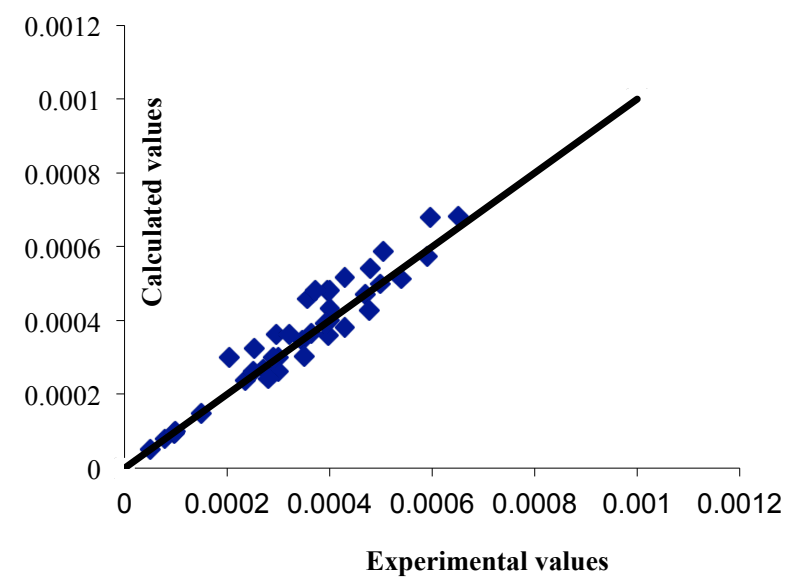

Fig 7: $\mathrm{K}_{\text {od }}$ Experimental vs. $\mathrm{K}_{\text {od }}$ calcultaed

Figure 7 shows the experimental of mass transfer coefficient of single liquid drops during formation versus the predicted values from the suggested model. The difference fit between the data and the prediction from the equations fall within the range of experimental error. When we compared the experimental results with values calculated based on the above equation a good agreement was found (34-37). As a conclusion, it can be stated that the suggested equation can be used for the correlation of the observed data when the down flow of the continuous phase greater than or 
equal zero i.e. in the presence and absence of down flow of the continuous phase. As mentioned earlier, to use the different model for mass transfer, one use the system properties and drop diameter to calculate the drop formation mass transfer. The observed mass transfer is considerably higher than the theoretically predicted values and this may be due to internal circulation caused by impinging jet while drop grows, interfacial turbulence, influence of the rest drop remaining after detachment and disturbance associated with drop detachment [16] . Parameter such as density, viscosity, drop diameter, interfacial tension, system property or impurities (if present) may also affect the value of mass transfer.

\subsection{The suggested model vs. the other models}

The experimental mass transfer coefficients during drop formation have been calculated for the surface stretch and the fresh surface element models and is compared with the suggested theoretical on the basis of the concentration driving force present in the drop at the start of the formation period (figure 7,8 ).

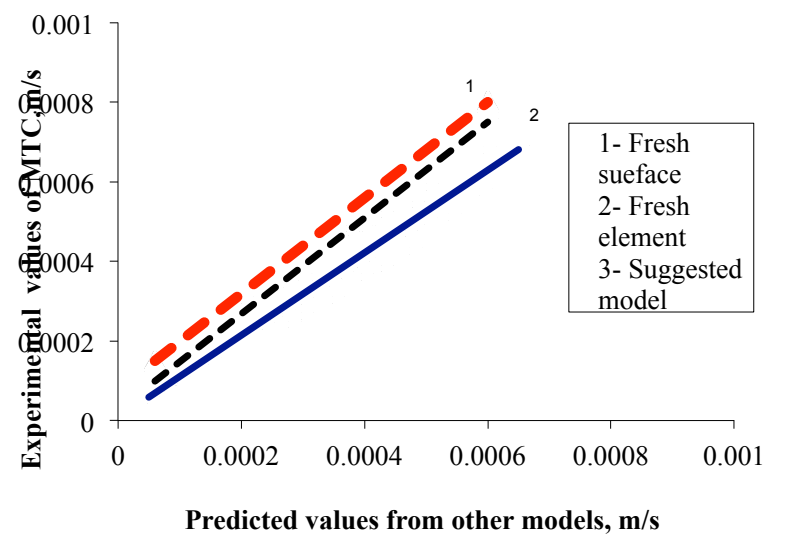

Fig. 8: The suggested model Vs.other models for drop mass transfer

No specific comparison have been made to other models since the prediction from the other models for drop formation is lower than that of the fresh surface elements model as should be expected of a more general form of the surface stretch model. On comparing results it is clear that good agreement between the experimental and predicted values has been obtained although the experimental values slightly higher than the predicted. The reason for this discrepancy is probably results of the hydrodynamic disturbances induced during the drop formation. Moreover most of the scatter can be attributed to neglected effect of circulation on the mass transfer during drop formation and also the difference in the physical properties between systems.

\section{Conclusion}

The results revealed that the observed mass transfer coefficients are largest in the initial stages of drop formation when convection is most significant. The mass transfer coefficient and the degree of extraction increase with increasing the down flow rate of the continuous phase. Both flow rate of flow has a significant effect on the rate of the mass transfer and the convection caused by the dispersed phase flow is more important than continuous phase. It is concluded that $30 \%$ of mass transfer occurred during drop formation period. Quantitative methods for prediction of mass transfer rates between the exposed surface of liquid droplet and the continuous phase flowing around the droplets are indispensable for the design of liquid-liquid extraction equipment. In this comparative study, two different models were tested i.e. surface stretch model and fresh surface element. A new model for calculating the mass transfer was suggested and compared with the available model in the literature. The results are in qualitative agreement with observation of other investigators but the experimental values of mass transfer coefficient were found greater than that predicted from the other models. There is a risk in going from lab bench data to a full-scale plant without the intermediate piloting stage. More direct comparison data for other liquid-liquid systems is evidently needed, together with a wide range of physical properties. Work is currently in progress at AVT Aachen- Germany, for studying other chemical systems that recommended by EFCE.

\section{Nomenclature}

d Drop diameter (m)

E Degree of extraction during formation period,

$(\mathrm{Ci}-\mathrm{C}$ o $) /\left(\mathrm{Ci}-\mathrm{C}^{*}\right)$

F Down flow of the continuous phase $(1 / \mathrm{min})$

$\mathrm{kd} \quad$ Dispersed phase mass transfer coefficient $(\mathrm{m} / \mathrm{s})$

tf, Drop formation time, (s)

Vd Volume of drop $\left(\mathrm{m}^{3}\right)$

\section{Greek letters}

$\begin{array}{ll}\mu & \text { Viscosity }(\mathrm{kg} / \mathrm{m} \mathrm{s}) \\ \rho & \text { Density }\left(\mathrm{kg} / \mathrm{m}^{3}\right) \\ \gamma & \text { Interfacial tension }(\mathrm{mN} / \mathrm{m}) \\ \text { Groups } & \\ \mathrm{Fr} & \text { Froude number, }(\mathrm{N} 2 \mathrm{D} 2 / \mathrm{g}) \\ \mathrm{Oh} & \text { Ohnesorge number }(\mu \mathrm{d} /(\rho \mathrm{cd} \gamma) 0.5 \\ \text { Ret } & \text { Turbine Reynolds number, }(\rho \mathrm{N} \mathrm{Dd} / \mu \mathrm{d}) \\ \text { Shd } & \text { Sherwood number, }(\mathrm{k} \mathrm{d} / \mathrm{D}) \\ & \\ \text { Subscripts } & \\ \mathrm{c} & \text { Continuous phase } \\ \mathrm{d} & \text { Dispersed phase } \\ \mathrm{f} & \text { Formation }\end{array}$

\section{Acknowledgements}

Mohsen A Hashem, working at RWTH Aachen University, as a visiting professor for six months on a grant from the Egyptian Government.

\section{References}

1. Gross-HardtE, E. Slusanschi, H. M. Bücker, A. Pfennig, and C. H. Bischof. Practical Shape Optimization of a Levitation Device for Single Droplets. Optimization and Engineering, 2008.
2. Gross-Hardt, E., A. Amar, S. Stapf, A. Pfennig, and B. Blümich. Flow Dynamics Inside a Single Levitated Droplet. Ind. \& Eng. Chem. Res., 1:416-423, 2006.

3. Clift, R.; Grace, J. R.; Weber, M. E. Bubbles, Drops, and Particles; Academic Press: New York, 1978. 
4. Treybal, R. Liquid extraction, McGraw-Hill Book Company, 1963

5. Gross-Hardt, E.; Henschke, M.; Klinger, S.; Pfennig, A. Design of pulsed extraction columns based on lab-scale experiments with a small number of drops. In Proceedings of the International Solvent Extraction Conference, ISEC 2002; Sole, K. C., Cole, P. M., Preston, J. S., Robinson, D. J., Eds.; 2002.

6. Groß-Hardt, E. M. Henschke, S. Klinger, and A. Pfennig. Design of pulsed extraction columns based on lab-scale experiments with a small number of drops. ISEC 2002.

7. Waheed, M. A.; Henschke, M.; Pfennig, A., 45, 4507-4514, Int. J. Heat Mass Transfer 2002

8. Gross-Hardt, E.; Henschke, M.; Pfennig, A., 49, 1611., AIChE J. 2003

9. Henschke, M. and A. Pfennig. Influence of Sieve Trays on the Mass Transfer of Single Drops. AIChE Journal, 48(2):227-234, 2002

10. Thornoton, J. D., ed., The Science and Practice of Liquid-Liquid Extraction, Oxford University Press, Oxford, 1992.

11. Hines, L. A. and Maddox, R.N., Mass transfer Fundamental and Application,Prentic -Hall, Inc 1985

12. Slater, M. J., Baird, M. H. I. and Liang, T. B., Chem. Eng. Sci., vol. 43, No. 2, pp 233-245, 1988.

13. Godfrey, J. C., and Slater, M. J, Ch.. 4 in Liquid Extraction Equipment, eds. by Slater, M. J. and Godfery, J. C., Pub, J. Wiley and Sons, 1994.

14. Hashem, M. A. Hashem, PhD Thesies, Bradford (UK) and ElMinia (Egypt) 1997

15. Hashem, M. A. and El-bassuoni, A., Theoretical Foundation of Chemical Engineering Journal, vol. 41, No. 5 pp. 530-535. 2007

16. Hashem, M. A., Proceeding of 6th International Conference RETBE'2006, Faculty of Eng. Alexandria University, Alexandria Egypt 18-20 Dec. 2006.

17. Hashem, M.A. and Slater, M.J., Proceeding of Fluid Mixing 5 Conference organized by the Inst. of Chem. Eng., University of Bradford, 4-5 July 1996
19. Hashem, M. A. and El-Bassouni A.A, The first International Conference for Multiphase Flow 2001, 14-16 March 2001 Orlando, Florida, USA.2001

20. Hashem, M. A, Alex Eng. J., Vol. 44. No.3, 2005

21. Misek, T., Berger, J., Schroter, J., EFCE Pub. Series No. 46, London 1985

22. Grace, J. R., Wairegi, T. H. and Nguyen, T. H., Trans. IChmE., 54,1976

23. Sedahmed, G. H., El-Kayar, A.M., Farag, H. A. and Noseir, S.A., The Chemical Engineering Journal, 62, 61-65, 1996..

24. Bailes, P. J., Gledhill, J., Godfrey, J. C. and Slater, J. M., Chem. Eng. Res. Des., vol. 64, January 1986.

25. Henschke, M. and Pfennig A. AIChE J., vol. 45, no. 10, ppf.2079, October 1999

26. Laddha, G,S, and Degaleesan, T.E., Transport Phenomena in Liquid Extraction, Tata McGraw-Hill Publishing Co. Ltd. 1976

27. Zimmerman, V. Halwachs, W. and Schugerl, K. ISEC 80 LiegeBelgium 6-12 September 1980

28. Schott R and Pfennig, Molecular physics, vol. 102, no.4, ppf331, February 2004

29. Heertjes, P.M., Holve, V.A. and Talsma H., Chem. Eng. J., 31, 483-494, 1954

30. Henschke, M. and Pfennig, A., AIChE J., vol. 48, no. 2, ppf. $227-$ February 2002

31. Liang, T.B. and Slater, M.J., Chem. Eng. Sci. 45(1), pp.97-105, 1990

32. Skelland, A.H.P.and Moeti, L.T. Ind. Eng. Chem. Res., 29, 22582267, 1990

33. Humphrey, J.A.C., Hummel, R.L. and Smith, J.W., Chem. En. Sci., 29, pp. 1496-, 1974

34. Godfrey, J.C. and Slater, M. J. ed, Liquid- Liquid Extraction Equipment, John Wiley and Sons Ltd. 1994

35. Marr, R. and Moser, F. Chem. Ing. Tech., 48(9), p.801, 1976.
18. Hashem, M. A., Proceeding of 3rd International Conference RETBE'2000, Faculty of Eng. Alexandria University, Egypt 18-20 Nov. 2000 